\title{
Implement Embedded Controller using FPGA Chip
}

\author{
Dr. Haresh Pandya ${ }^{1}$, Mr. Mahesh Rangapariya ${ }^{2}$, Mr. Jitendra Rajput ${ }^{3}$ \\ Professor \& Head, Department of Electronics, Saurashtra University, Rajakot, Gujrat, India ${ }^{1}$ \\ Ph.D. Student \& Visiting Lecturer, Department of Electronics, Saurashtra University, Rajakot, Gujarat, India ${ }^{2}$ \\ Assistant Professor of Electronics at SSR College of Science, (Affiliated to Pune University), Silvassa, D \& NH, India ${ }^{3}$
}

\begin{abstract}
The designer of an FPGA embedded processor system has complete flexibility to select any combination of peripherals and controllers. In fact, the designer can invent new, unique peripherals that can be connected directly to the processor bus. If a designer has a non-standard requirement for a peripheral set, this can be met easily with an FPGA embedded processor system. For example, a designer would not easily find an off-the-shelf processor with ten UARTs. However, in an FPGA, this configuration is very easily accomplished.
\end{abstract}

Keyword: Embedded Controller, VHDL (VHSIC Hardware Description Language), FPGA (Field Programmable Gate Arrays), Electronics Switch.

\section{INTRODUCTION}

FPGAs are increasingly used to implement embedded digital systems because of their low time-to-market and low costs compared to integrated circuit design, as well as their superior performance and area over a general purpose microprocessor. However, the hardware design necessary to achieve this superior performance and area is very difficult to perform causing long design times and preventing wide-spread adoption of FPGA technology. The amount of hardware design can be reduced by employing a microprocessor for less-critical computation in the system. Often this microprocessor is implemented using the FPGA reprogrammable fabric as a soft processor which can preserve the benefits of a single-chip FPGA solution without specializing the device with dedicated hard processors. Current soft processors have simple architectures that provide performance adequate for only the least-critical computations. Embedding a processor inside an FPGA has many advantages. Specific peripherals can be chosen based on the application, with unique user-designed peripherals being easily attached. A variety of memory controllers enhance the FPGA embedded processor system. interface capabilities. FPGA embedded processors use general-purpose FPGA logic to construct internal memory, processor busses, internal peripherals, and external peripheral controllers (including external memory controllers). Soft processors are built from general-purpose FPGA logic as well. As more pieces (busses, memory, memory controllers, peripherals, and peripheral controllers) are added to the embedded processor system, the system becomes increasingly more powerful and useful. However, these additions reduce performance and increase the embedded system cost, consuming FPGA resources. Likewise, large banks of external memory can be connected to the FPGA and accessed by the embedded processor system using included memory controllers. Unfortunately, the latency to access this external memory can have a significant, negative impact on performance.

FPGA manufacturers often publish embedded processor performance benchmarks. Like all other companies running benchmarks, these FPGA manufacturers purposely construct and use an FPGA embedded processor system that performs the best for each specific benchmark. This means that the FPGA processor system constructed to run the benchmark has very few peripherals and runs exclusively using internal memory. The embedded designer must understand how a real-world designs. Specific peripheral set and memory architecture will affect the system performance. However, no easy formula or chart exists showing how to compare the performance and cost for different memory strategies and peripheral sets.

\section{Advantages of an FPGA embedded processor}

An FPGA embedded processor system offers many exceptional advantages compared to typical microprocessors including:

1) Customization

2) Obsolescence mitigation

3) Component and cost reduction

4) Hardware acceleration

\section{Hardware acceleration}

Perhaps the most compelling reason to choose an FPGA embedded processor is the ability to make tradeoffs between hardware and software to maximize efficiency and performance. If an algorithm is identified as a software bottleneck, a 
custom co-processing engine can be designed in the FPGA specifically for that algorithm. This co-processor can be attached to the FPGA embedded processor through special, low-latency channels, and custom instructions can be defined to exercise the co-processor. With modern FPGA hardware design tools, transitioning software bottlenecks from software to hardware is much easier since the software $\mathrm{C}$ code can be readily adapted into hardware with only minor changes to the $\mathrm{C}$ code.

\section{Component and cost reduction}

With the versatility of the FPGA, previous systems that required multiple components can be replaced with a single FPGA. Certainly this is the case when an auxiliary I/O chip or a co-processor is required next to an off-the-shelf processor. By reducing the component count in a design, a company can reduce board size and inventory management, both of which will save design time and cost.

\section{Obsolescence mitigation}

Some companies, in particular those supporting military contracts, have a design requirement to ensure a product lifespan that is much longer than the lifespan of a standard electronics product. Component obsolescence mitigation is a difficult issue. FPGA soft-processors are an excellent solution in this case since the source HDL for the soft-processor can be purchased Ownership of the processor. VHDL code may fulfill the requirement for product lifespan guarantee.

\section{FLOW CHART OF FPGA EMBEDDED SYSTEM}

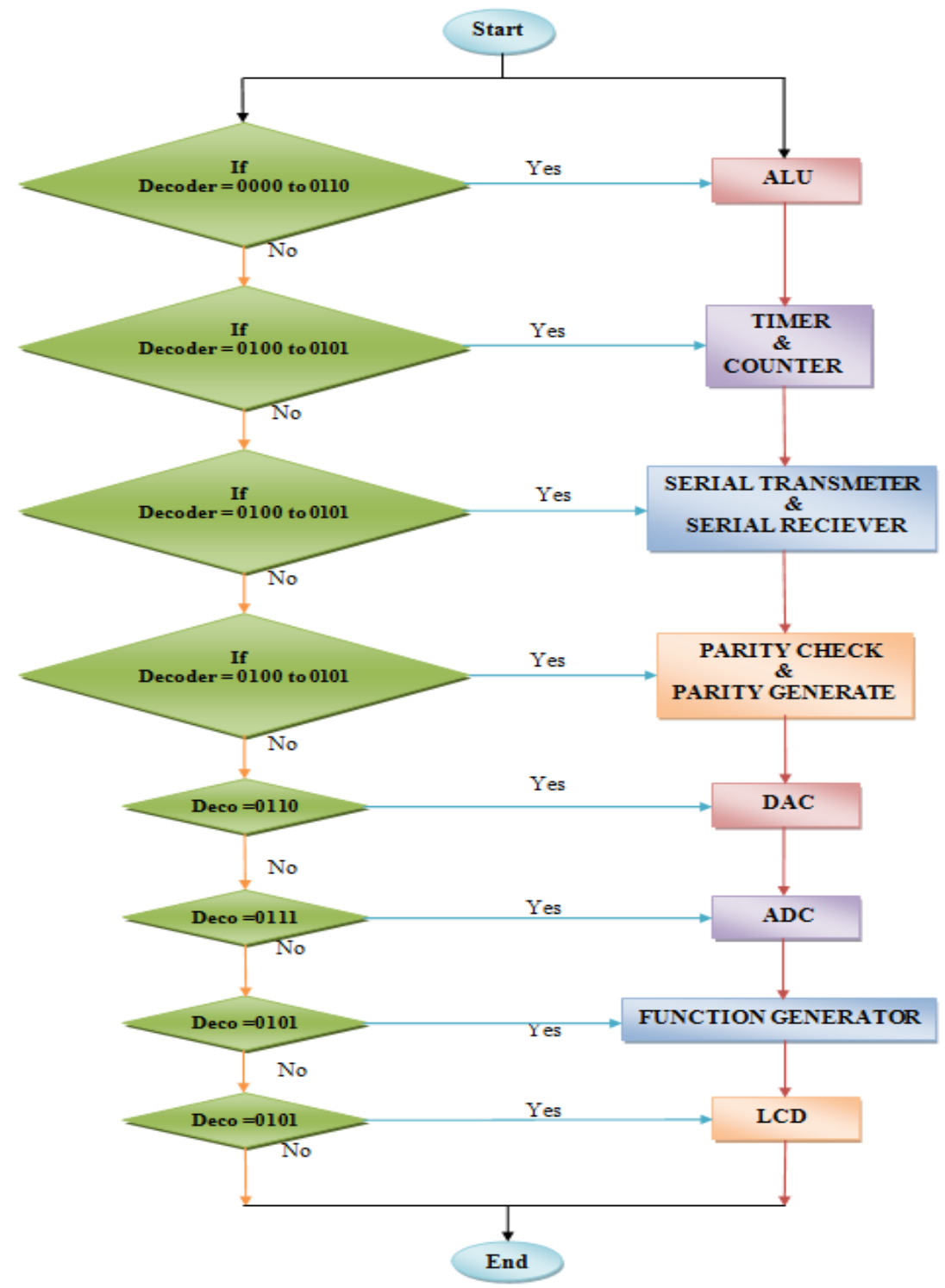




\section{BLOCK DIAGRAM OF FPGA EMBEDDED SYSTEM}

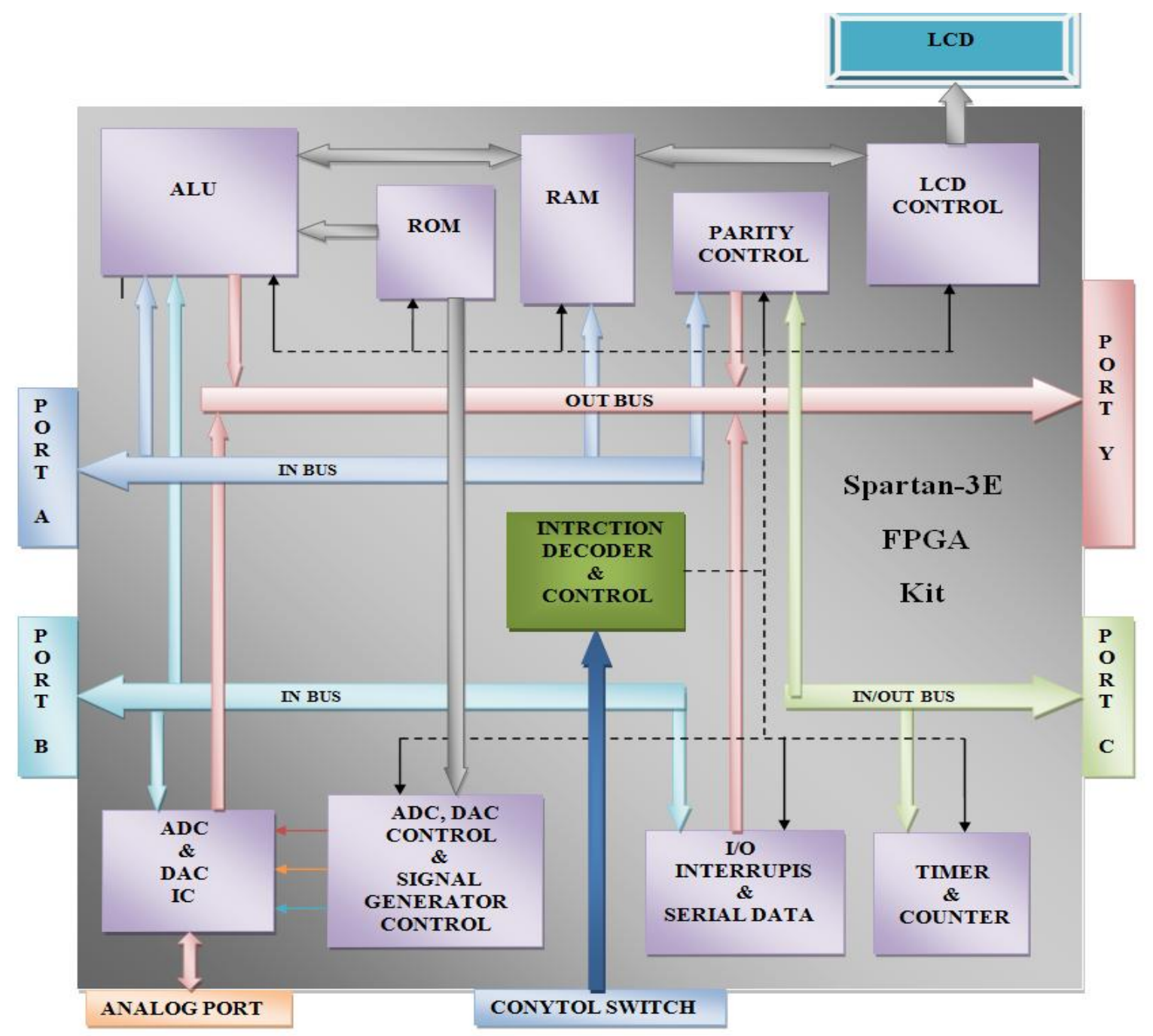

Block Diagram description

The microcontroller has a following main block. Like ALU, Control Unit, Instruction Decoder, in built RAM/ROM, I/O Port, Data bus/Control bus, Timer, Counter, Serial Transmitter/Receiver and Interrupt control. Hardware of the entire block is fix and limited so, Working of block is specific. For Embedded application external ADC, DAC connect with microcontrollers therefore, district Sensor assembly and ADC/DAC externally connection require.

Now a day's FPGA base embedded board is available. Here Xilinx Spartan 3E FPGA board use for design of embedded controller. Xilinx kit has a following facility like, on board two channels serial ADC, four channels serial DAC, 16X2 LCD, 8-Switch and 8-LED. ALU, Control Unit, Instruction Decoder, in built RAM/ ROM, I/O Port, Data bus/Control bus, Timer, Counter, Serial Transmitter/Receiver and Interrupt control block make inside the FPGA using VHDL program. Above all block are reprogrammable so, functionality of all block modify using VHDL program.

Using VHDL program $4 \mathrm{bit} / 8 \mathrm{bit} / 16 \mathrm{bit} / 32 \mathrm{bit} / 64 \mathrm{bit} / 128 \mathrm{bit} . . . .$. ALU easily create in side FPGA logic cell. Function of control unit is totally set using VHDL task. Design and modification of instruction set depends VHDL program so, we create over personal mnemonics. Four data bus create they are connected to port A, port B, port C and port Y. All the bus is internally connected to each block of embedded controller.

\section{ALU}

ALU give three arithmetic operations and five logical operations. Arithmetic operations are addition, subtraction and multiplication. Logic operations are NOT, OR, AND, EXOR and EXNOR. Here 4-Bit ALU block is design using VHDL program so, operation and bit width of ALU is change using program.

\section{MEMORY}

Memory of embedded controller is design inside FPGA chip. RAM/ROM create using VHDL program so, memory size and bit width of both is change using program. VHDL has a three data object and four data type. Data object are 
variable, signal, constant and data type are single, double, vector and array. Using constant, vector are array make ROM. Using signal, vector and array make a RAM. VHDL support integer data type so, memory address is decimals number. Using decimal address method, memory location is easily access. VHDL base memory single pin use for read and write operation.

\title{
LCD CONTROL
}

Control and DATA signal are use to create character on display. Three main handshake signals are as follow EN, RS, RW and DATA. LCD has a two operation mode one initialization and second display. LCD control block has an initialization and operation mode VHDL program. LCD initialization and operation program store in LCD ROM they execute location by location. First execute initialization program after that execute operation program character bit. As per the applying of character bit character is display on screen.

\section{TIMER/COUNTER}

Normally, microcontroller in built timer works as timer or counter. Really, fact that both are not operating simultaneously. In FPGA kit up/down timer and up/down counter is create using VHDL program. FPGA base two Timers and two counters are creating and they are increment/decrement type. All timer and counter work independence simultaneously. VHDL signal object and arithmetic operator is use to make timer and counter. Timer and counter has a control bit they also use to generate soft interrupt. FPGA embedded microcontroller Port $\mathrm{C}$ use for timer and counter.

\section{SERIAL TRANSMETER\&RECEIVER}

Microcontroller 8051 has an only one in built serial transmitter and receiver. FPGA base microcontroller more than one serial transmitter/receiver designed and all work indecently to each author. Serial transmitter consists of parallel to serial conversion program and serial receiver consists of serial to parallel conversion program. Serial clock, Information data and Protocol bit are create using VHDL program so, change using program. Serial transmitter/receiver has a control bit they also use to generate soft interrupt. FPGA embedded microcontroller Port C use for serial transmitter and receiver.

\section{INTERRUPT CONTROL}

In FPGA embedded microcontroller Port $\mathrm{C}$ use for hardware interrupt. Port $\mathrm{C}$ two pin use for timer/counter, two pin use for serial communication and remaining four pin use for hardware interrupt. Priority of all interrupt set using VHDL program and change it, if require. Hardware and software both interrupt work independence simultaneously. For hardware interrupt level sensitive and positive/negative edge (trigger) sensitive both are create. Software control like, timer interrupt, serial communication interrupt are also control using this block.

\begin{abstract}
ADC/DAC CONTROL
ADC/DAC both required handshaking signal to control all operation. Control sequence of ADC and DAC are different so, control block internally divided into two parts. ADC control part generate ADC control signal and DAC control part generate DAC control signal. ADC require five control signal as like, spi_mosi, amp_cs, spi_sck, amp_shdn and ad_conv. DAC also require five control signals like, spi_mosi, dac_cs, spi_sck, dac_clr and spi_miso. Above all signal is produce by using VHDL program with proper sequence. ADC and DAC both are work in serial input mode so, some time input data also allaying using VHDL program.
\end{abstract}

\section{SIGNAL GENERATOR CONTROL}

FPGA base signal generator consists of VHDL ROM and DAC. Different weighted digital data permanent store inside VHDL ROM. Address control program continue and sequentially generate different location of memory. According to generated address, memory location is select and DAC continue read digital data from ROM. DAC produce different analog output according to input digital data. Value of analog output is very with different time function.

\section{VHDL PROGRAMM FOR FPGA EMBEDDED SYSTEM}

\footnotetext{
-- Company:

-- Engineer:

-- Create Date: 19:20:02 01/20/2008

-- Design Name:

-- Module Name: ECP - Behavioral

-- Project Name:

-- Target Devices:

-- Tool versions:

-- Description:

-- Dependencies:

-- Revision:

-- Revision 0.01 - File Created
} 
UGC Approved Journal

-- Additional Comments:

library IEEE;

use IEEE.STD_LOGIC_1164.ALL;

use IEEE.STD_LOGIC_ARITH.ALL;

use IEEE.STD_LOGIC_UNSIGNED.ALL;

---- Uncomment the following library declaration if instantiating

---- any Xilinx primitives in this code.

--library UNISIM;

--use UNISIM.VComponents.all;

entity ECP is

GENERIC $(\mathrm{n}:$ INTEGER $:=3 ; \mathrm{m}:$ INTEGER $:=2 ; \mathrm{r}:$ INTEGER $:=7$ );

Port ( rst,bclk,pro,exi,cnt_in,dec : in STD_LOGIC;

miso,shdn,ampad,ampdo,mosi1 : in STD_LOGIC;

RS,E,RW,clk,clk1,daccs,ampcs,adcon : inout STD_LOGIC; ssb,ceo,initb,last : out STD_LOGIC;

ashdn, mosi,A_Dclk,DAC_clr : out STD_LOGIC;

a,b,c,alu_data : inout STD_LOGIC_VECTOR ( $\mathrm{r}$ downto 0); deco : inout STD_LOGIC_vector (4 downto 0);

digi_in : in STD_LOGIC_vector $(10$ downto 0$)$;

y : out STD_LOGIC_vector (15 downto 0);

end ECP;

DB1 : inout STD_LOGIC_VECTOR (4 downto 0));

architecture Behavioral of sd is

$\begin{array}{ll}\text { signal btemp } & : \text { integer range } 0 \text { to } 500000:=0 ; \\ \text { signal btemp } 1 & \text { : integer range } 0 \text { to } 4800000:=0 ;\end{array}$

signal btemp1 : integer range 0 to $4800000:=0$
signal add,count 0 : integer range 0 to $31:=0 ;$

-----ALU------

signal tempa,tempb : STD_LOGIC_VECTOR(r downto 0);

signal add_y,sub_y,not_y, or_y, and_y,xor_y,xnor_y: STD_LOGIC_vector(0 to r);

signal mlt_y : STD_LOGIC_VECTOR(0 to 15$)$;

----TIMER/COUNTER----

signal ti_dwn_out,ti_up_out,cont_out : STD_LOGIC;

constant timer_up_mod :integer range 0 to $10:=0 ;---\bmod --0=>50 \%---\bmod --1=>25 \%--\bmod --2=>10 \%--\bmod --3=>5 \%$ constant timer_dwn_mod :integer range 0 to $5:=2$; ---mod-- $0=>50 \%---\bmod --1=>25 \%--\bmod --2=>10 \%--\bmod --3=>5 \%$ signal timer0,ti_up_duty,timer1,ti_dwn_duty : integer range 0 to 100 ;

signal cnt_data,cnt1_data : STD_LOGIC_VECTOR (4 downto 0$)$;

signal SE_cs2,SE_cs1,se_in,seri_out: STD_LOGIC ;

signal pa_data,pa_in : STD_LOGIC_VECTOR(r downto 0);

signal h1 : STD_LOGIC_VECTOR (0 to 9);

signal h2 : STD_LOGIC_VECTOR $(0$ to 7$)$

signal temp1 : integer range 0 to $10:=0 ;$

signal temp2 : integer range 0 to $7:=0$;

signal temp3 : integer range 0 to $12:=0$;

signal temp4 : integer range 0 to $12:=0$

signal parity_bit : STD_LOGIC;

signal parity : STD_LOGIC_VECTOR(r downto 0);

signal prt_data,a1: STD_LOGIC_VECTOR(r downto 0);

signal DAC_SET,DAC_cs1,DAC_cs2: STD_LOGIC;

----DAC-----

signal h3 :STD_LOGIC_VECTOR $(23$ downto 0$)$;

signal h4 :STD_LOGIC_VECTOR $(15$ downto 0$)$

signal DAC_in : STD_LOGIC_VECTOR (15 downto 0$)$;

signal DACtemp1 : integer range 0 to $26:=0$;

signal DACtemp2 : integer range 0 to $23:=0$;

signal smiso,AMP SET,ADC_cs1,ADC_cs2: STD_LOGIC;

signal ADC_data : STD_LOGIC_VECTOR(13 downto 0);

signal h5 $\quad$ : STD_LOGIC_VECTOR $(7$ downto 0$)$;

signal h6 : STD_LOGIC_VECTOR (0 to 35);

signal y1 :STD_LOGIC_VECTOR (0 to 15);

signal ADCtemp1 : integer range 0 to $10:=0$; 
UGC Approved Journal

signal ADCtemp2 : integer range 0 to $8:=0$; signal ADCtemp3 : integer range 0 to $34:=0$; signal ADCtemp4 : integer range 0 to $32:=0$;

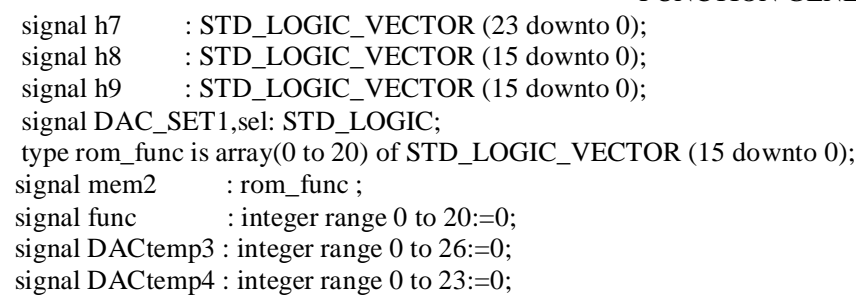

$\operatorname{mem}(0)<=$ "00000" ;

$\operatorname{mem}(1)<=$ "00010" ;--funct

$\operatorname{mem}(2)<=$ "01000" ;

$\operatorname{mem}(3)<=$ "00000" ;--entry $\bmod$

$\operatorname{mem}(4)<=$ "00110" ;

$\operatorname{mem}(5)<=$ "00000" ;--display on

$\operatorname{mem}(6)<=$ "01100" ;

$\operatorname{mem}(7)<=$ "00000" ;--clear display

$\operatorname{mem}(8)<=$ "00001" ;

$\operatorname{mem} 1(0)<=$ "00000" ;--clear display

$\operatorname{mem} 1(1)<=$ "00001" ;

mem1 $(2)<=$ "01000" ;-- GIVE 1-LINE-ADDRESS

$\operatorname{mem} 1(3)<=$ "00110" ;

$\operatorname{mem} 1(4)<=$ "10100" ; -- L

$\operatorname{mem} 1(5)<=$ "11100" ;

$\operatorname{mem} 1(6)<=" 10100 "$; -- C

$\operatorname{mem} 1(7)<=" 10011 "$;

$\operatorname{mem} 1(8)<=" 10100 " ;--\mathrm{D}$

$\operatorname{mem} 1(9)<=" 10100 "$;

$\operatorname{mem} 1(10)<=$ "01100" ;-- GIVE 2-LINE-ADDRESS

$\operatorname{mem} 1(11)<=$ "00100" ;

$\operatorname{mem} 1(12)<=$ "10100" ; -- C

$\operatorname{mem} 1(13)<=" 10011 "$;

mem1 $(14)<=$ "10100" ; -- O

$\operatorname{mem} 1(15)<=$ "11111" ;

$\operatorname{mem} 1(16)<=" 10100 " ;--\mathrm{N}$

$\operatorname{mem} 1(17)<=$ "11110" ;

mem1 $(18)<=$ "10101" ; -- T

$\operatorname{mem} 1(19)<=" 10100 "$;

$\operatorname{mem} 1(20)<=" 10101 " ;--\mathrm{R}$

$\operatorname{mem} 1(21)<=$ "10010" ;

$\operatorname{mem} 1(22)<=" 10100 "$; -- O

$\operatorname{mem} 1(23)<=" 11111 "$;

$\operatorname{mem} 1(24)<=$ "10100" ; -- L

$\operatorname{mem} 1(25)<=$ "11100" ;

$\operatorname{mem} 2(0)<=$ "0000000000000000" ;

$\operatorname{mem} 2(1)<=$ "0000001000000000" ;

$\operatorname{mem} 2(2)<=$ "0000000100000000" ;

$\operatorname{mem} 2(3)<=$ "0000000010000000" ;

$\operatorname{mem} 2(4)<=$ "0000000001000000" ;

$\operatorname{mem} 2(5)<=$ " $0000000000100000 "$ "

$\operatorname{mem} 2(6)<=$ "0000000000010000" ;

$\operatorname{mem} 2(7)<=$ "0000000000001000" ;

$\operatorname{mem} 2(8)<=$ "0000000000000100" ; 
UGC Approved Journal

$\operatorname{mem} 2(9)<=$ "0000000000000010" ;

$\operatorname{mem} 2(10)<=$ "0000000000000001"

mem2(11)<= "0000000000000010";

mem2(12)<= "0000000000000100" ;

$\operatorname{mem} 2(13)<=" 0000000000001000 "$;

$\operatorname{mem} 2(14)<=$ "0000000000010000" ;

mem $2(15)<=$ "0000000000100000" ;

mem2(16)<= "0000000001000000";

mem2(17)<= "0000000010000000" ;

mem2(18)<= "0000000100000000" ;

mem2(19)<= "0000001000000000" ;

$\operatorname{mem} 2(20)<=$ "0000010000000000" ;

$\mathrm{b}<=$ "00001111"; $\mathrm{a}<=$ "01010101"; deco(4)<= NOT dec ;

tempa $<=\mathrm{a}$; pa in $<=\mathrm{a}$; $\mathrm{a} 1<=\mathrm{a}$; tempb $<=\mathrm{b}$; se in $<=\mathrm{b}(0)$; DAC in $<=\mathrm{a} \& \mathrm{~b}$

$\mathrm{c}(7)<=\mathrm{ti}$.up_out; $\mathrm{c}(6)<=\mathrm{ti} \_\mathrm{dwn} \_$out; $\mathrm{c}(5)<=$ cont_out; $\mathrm{c}(4)<=$ seri_out; $\mathrm{c}(3)<=$ parity_bit; $\mathrm{c}(2)<=$ smiso;

--process $(\mathrm{Clk}, \mathrm{rst})$

-- begin

-- if(rst='1')then

-- add $<=0$;

-- elsif(Clk'event and $\mathrm{Clk}=$ ' 0 ')then

-- add $<=$ add +1 ;

--if(add=7)then

-- add $<=0$

-- end if;

--end if;

--addl<=conv_std_logic_vector(add,3);

-- if(pro='1')then

-- end if;

--if(pro='0')then

$$
\text { -- mem(add) }<=\text { da_in ; }
$$

$$
\text { --deco }<=\text { mem(add); }
$$

--end if;

-- decol $<=$ mem(add)

--end process;

-----board ckocl---50M--------

process(bclk)

begin

$$
\text { ----mem---- }
$$

btemp $<=$ btemp +1 ;

if $($ bclk'event and bclk = ' 0 ')then

$$
\text { clk<='1'; }
$$

if(btemp $>=250000)$ then

else

end process;

process(bclk)

$$
\text { begin }
$$$$
\mathrm{clk}<={ }^{\prime} 0 \text { '; end if; end if ; }
$$

btemp $1<=$ btemp $1+1$

clk1<='1';

$$
\text { if(btemp1>=2400000)then }
$$

else

end process;

clk1<=' 0 '; end if; end if ;

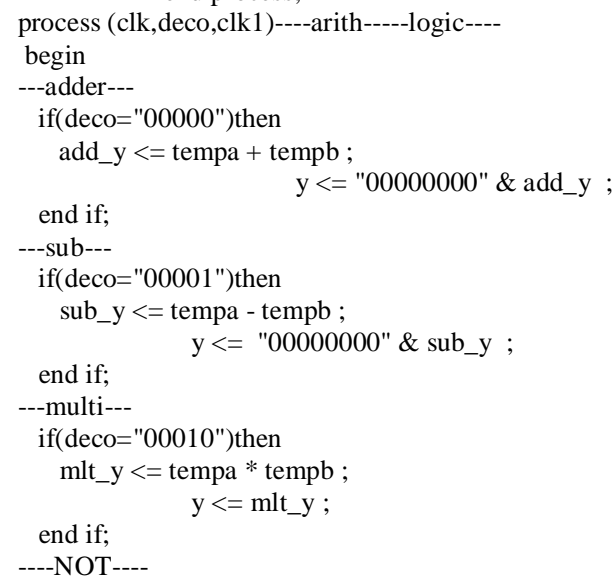


UGC Approved Journal

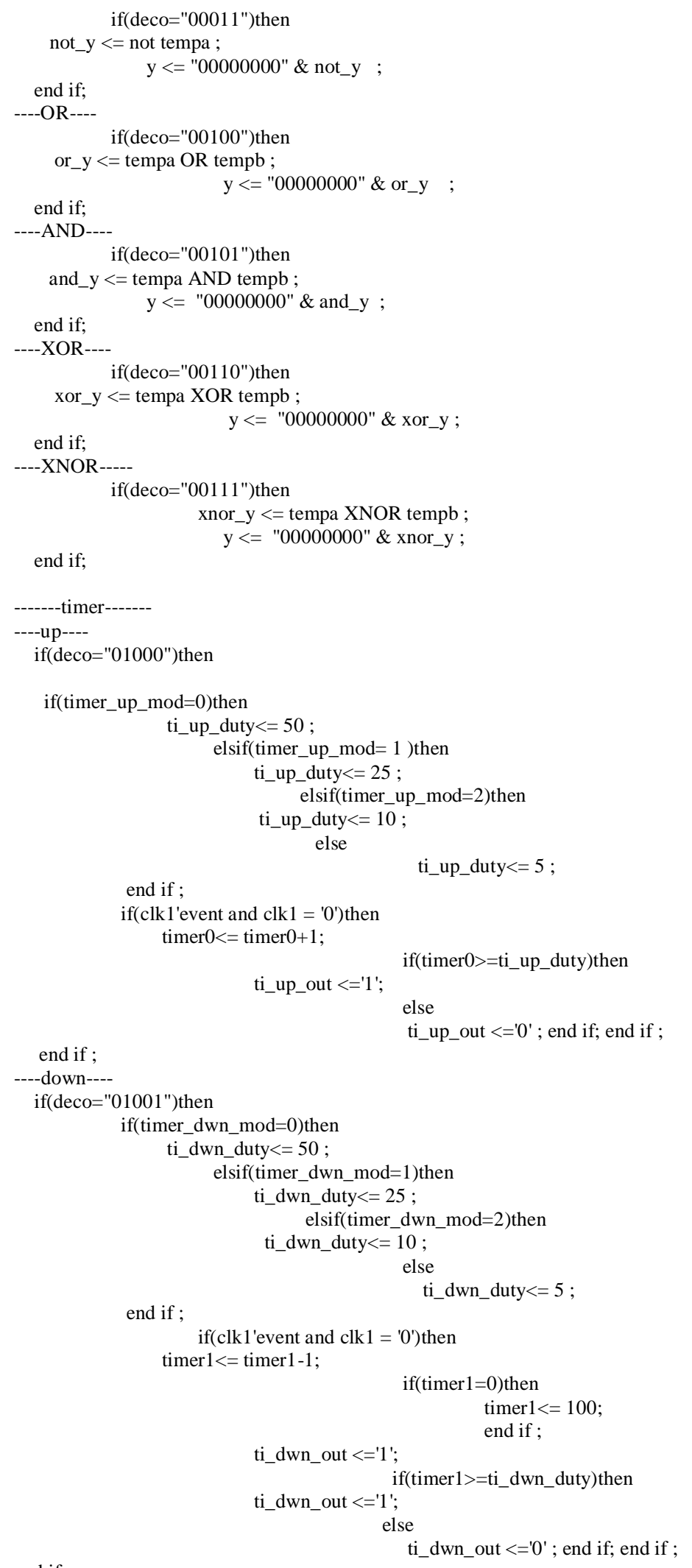

end if ;

----COUNTER----

if $(\mathrm{deco}=" 01010 ")$ then

---if(cnt_in'event and cnt_in= '0')then-----count---in-----

if $(\mathrm{clk} 1$ 'event and clk1 $=$ ' 0 ')then-----count---in----count $0<=$ count $0+1$; 
UGC Approved Journal

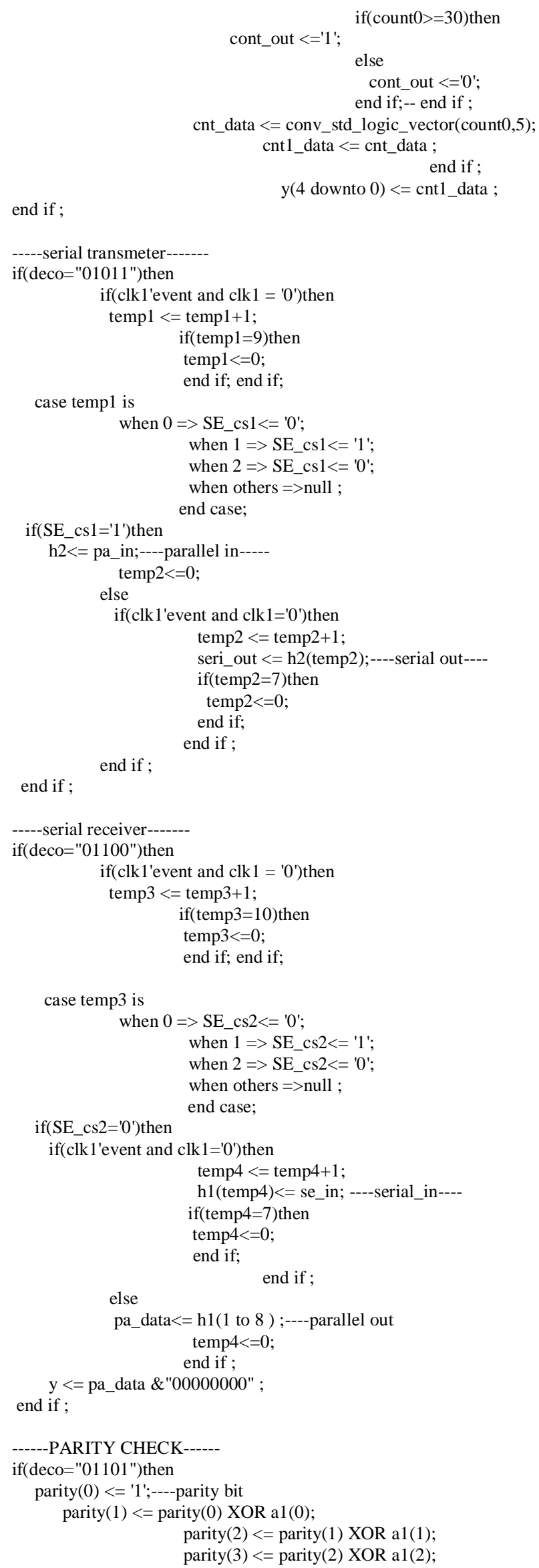




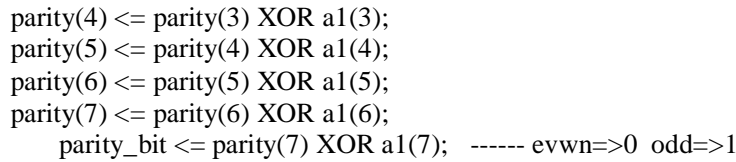

end if ;

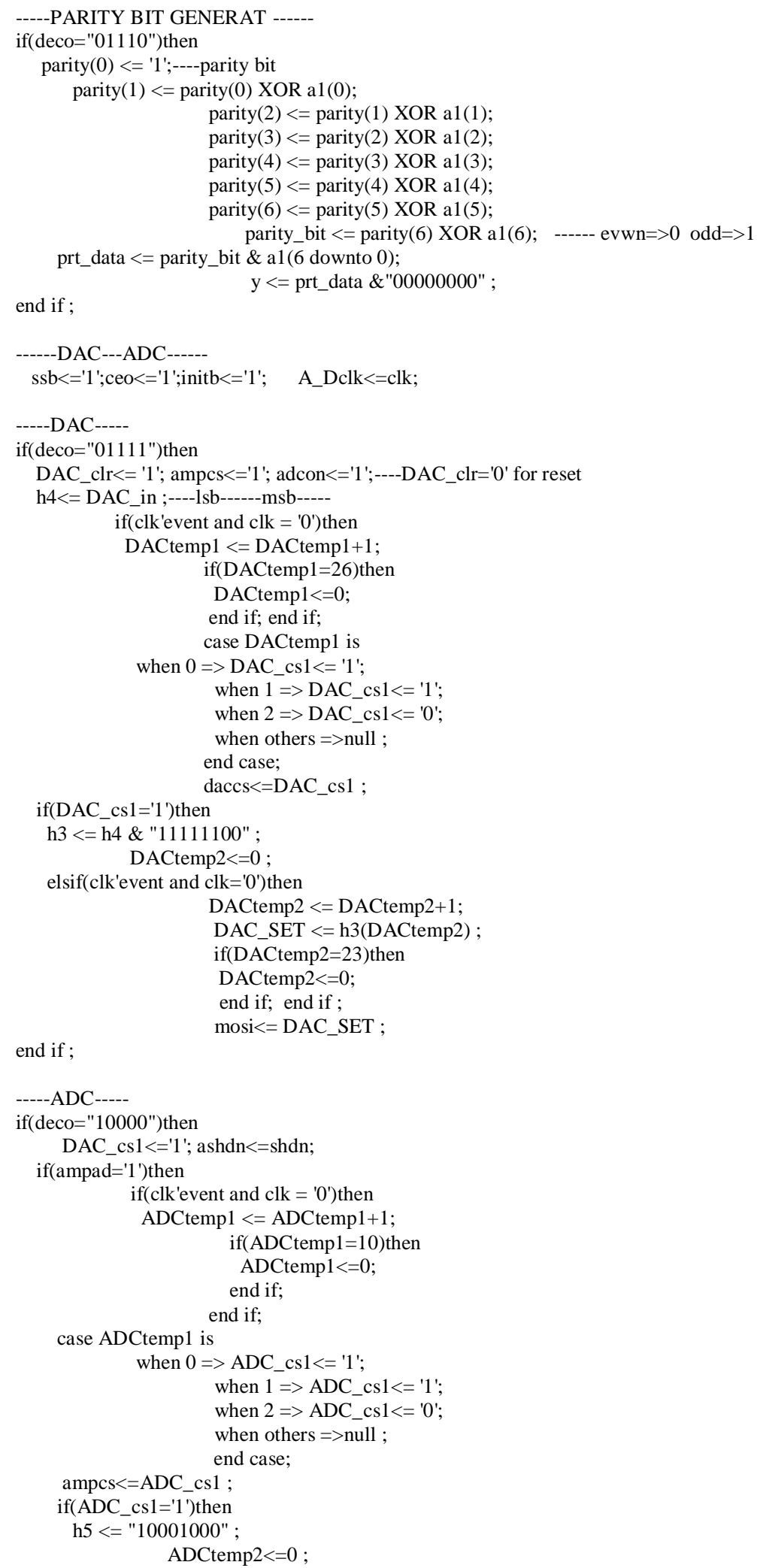


UGC Approved Journal

elsif(clk'event and clk='0')then

ADCtemp $<=$ ADCtemp2+1;

AMP_SET < $<$ h5(ADCtemp2) ;

if $($ ADCtemp2 $=8)$ then

ADCtemp $2<=0$;

end if ;

end if;

end if ;

if $\left(\operatorname{ampad}={ }^{\prime}\left(0^{\prime}\right)\right.$ then

mosi<= AMP_SET ;

if(clk'event and clk = '0')then

ADCtemp3 <= ADCtemp3+1;

if $($ ADCtemp3 34$)$ then

ADCtemp $3<=0$;

end if;

end if ;

end if;

case ADCtemp3 is

when $0 \Rightarrow$ ADC_cs $2<=$ ' 0 ';

when $1 \Rightarrow$ ADC_cs $2<=$ ' 1 ';

when $2 \Rightarrow$ ADC_cs $2<=$ ' 0 ';

when others $=>$ null ;

end case;

adcon $<=$ ADC_cs 2 ;

smiso<=miso;

if(ADC_cs $\left.2={ }^{\prime} 0^{\prime}\right)$ then

if(clk'event and clk='0')then

ADCtemp4 <= ADCtemp4+1;

h6(ADCtemp4)<= smiso ;

if(ADCtemp4=35)then

ADCtemp $4<=0$;

else

end if; end if ;

y1 <= h6(1 to 16$)$;---msb----1sb-----

ADCtemp $4<=0$;

end if ;

end if ;

$\mathrm{y}<=\mathrm{y} 1$;

-----FUNCTION GENERATOR-----

if $($ deco="10001")then

DAC_clr<= '1'; ampcs<='1'; adcon<='1';----DAC_clr='0' for reset

h9<= h8 ;----1sb------msb-----

if(clk'event and clk = '0')then

DACtemp3 <= DACtemp3+1;

if(DACtemp3=26)then

DACtemp $3<=0$;

end if; end if;

case DACtemp3 is

when $0=>$ DAC_cs $2<=$ ' 1 ';

when 1 => DAC_cs2<= '1';

when $2=>$ DAC_cs $2<=$ ' 0 ';

when others $=>$ null ;

end case;

daccs $<=$ DAC_cs 2 ;

if(DAC_cs2='1')then

h7 < = h9 \& "11111100" ;

DACtemp $4<=0$;

elsif(clk'event and clk='0')then

DACtemp4 <= DACtemp4+1;

DAC_SET1 <= h7(DACtemp4) ;

if(DACtemp4=23)then

DACtemp $4<=0$;

end if; end if ;

mosi<= DAC_SET1 ;

end if ;

if(DAC_cs2'event and DAC_cs2 = '0')then

func $<=$ func +1 ;

if $($ func $=20)$ then

last $<=$ ' 1 '

else

func $<=0$;

last $<=$ ' $^{\prime}$; 
UGC Approved Journal

end if ;

$\mathrm{h} 8<=$ mem 2 (func)

end if ;

end process;

-----LCD--CONTROL-----

process(clk,deco)

$$
\text { begin if(clk'event and clk }=\text { ' } 0 \text { ')then }
$$

$\operatorname{lcd} 1<=\operatorname{lcd} 1+1$

if $(\operatorname{lcd} 1>=2$ and $\operatorname{lcd} 1<=8)$ then

$\mathrm{RW}<=$ ' 0 ';

else

$\mathrm{RW}<=' 1$ ' ; end if; end if

if $($ clk'event and $\mathrm{clk}=$ ' 0 ')then

$\operatorname{lcd} 2<=\operatorname{lcd} 2+1$;

$\mathrm{E}<==^{\prime} 1$ if $(1 \mathrm{~cd} 2>=4$ and $1 \mathrm{~cd} 2<=6)$ then

else

end process;

$\mathrm{E}<=^{\prime} 0^{\prime}$; end if; end if ;

process $(\mathrm{RW}$, deco)
begin

if $(\mathrm{deco}=" 10010 ")$ then $\quad----$ DATA DISPLAY ----

if $\left(\mathrm{RW}\right.$ 'event and $\left.\mathrm{RW}=\mathrm{\prime}^{\prime} \mathrm{O}\right)$ then

$1 \mathrm{~cd} 4<=1 \mathrm{~cd} 4+1$;

$$
\begin{aligned}
& \operatorname{if}(\operatorname{lcd} 4=25) \text { then } \\
& \operatorname{lcd} 4<=0 ;
\end{aligned}
$$

end if ;

end if ;

else

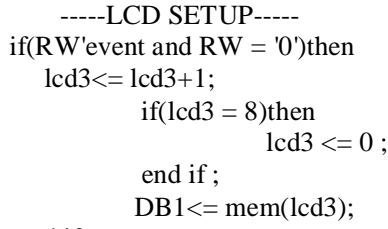

end if ;

end if ;

end process;

end Behavioral;

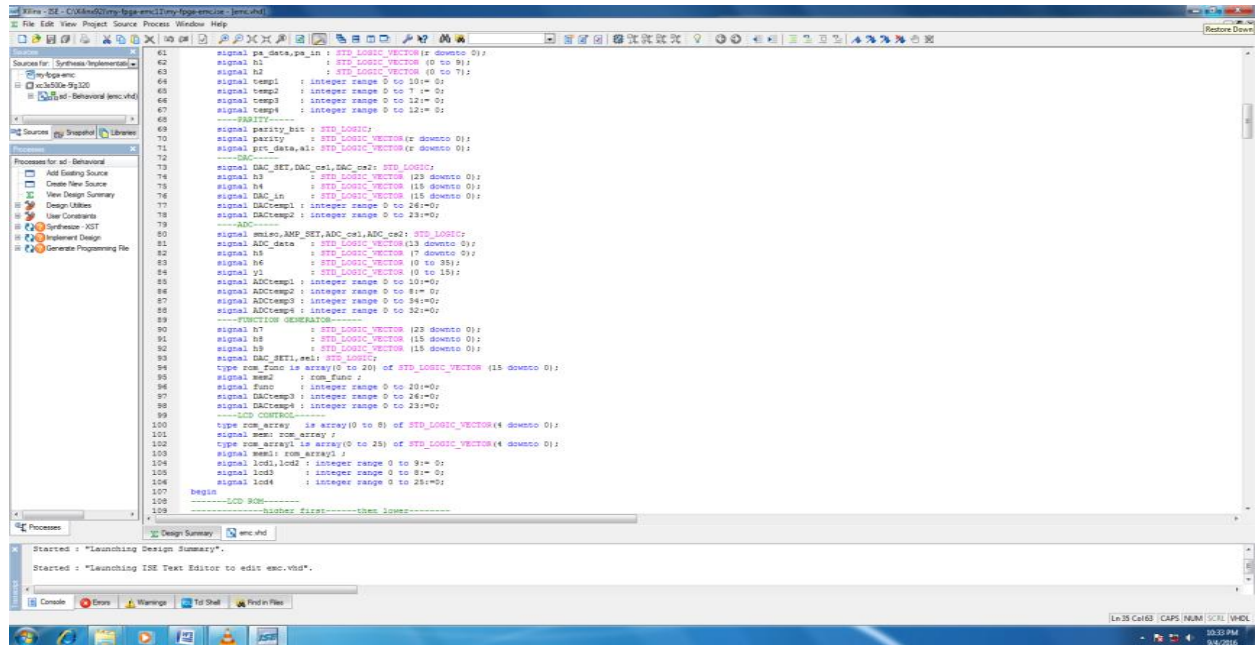

\section{SIMULATE BEHAVIORAL MODEL}

You can now run a functional simulation on the display drive module. With display_drive.vhd highlighted in the Source window, the Process window will give all the available operations for the particular module. A VHDL file can be synthesized and then implemented through to a bit stream. Normally, a design consists of several lower level modules wired together by a top-level file. In this instance, we are going to simulate only one lower-level module to introduce the functional simulation methodology. 
UGC Approved Journal

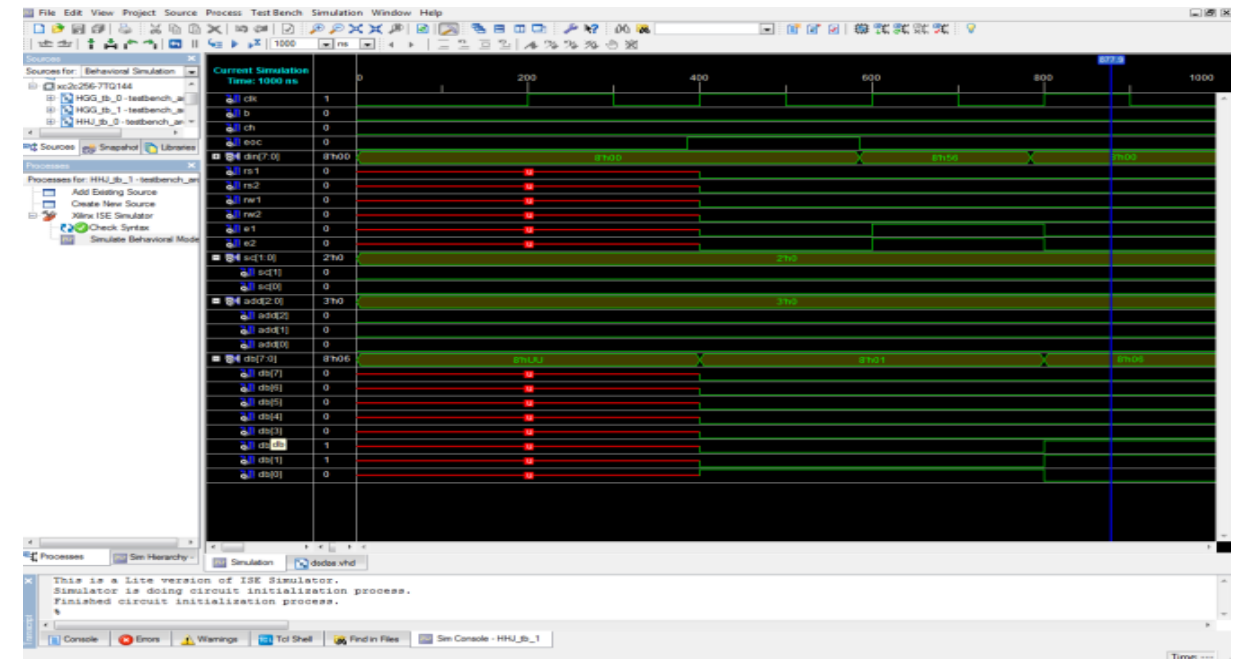

\section{REFERENCES}

[1] VHDL Programming by Example By Douglas L. Perry

[2] Circuit Design with VHDL By Volnei A. Pedroni

[3] DIDITAL DESIGN principles \& practices By JOHN F. WAKERLY

[4] Fundamentals of Digital Logic with VHDL Design. By Stephen Brownand Zvonko Vranesic

[5] The Practical Xilinx Designer Lab Book- Prentice Hall. By David Van den Bout

[6] The Practical Xilinx Designer Lab Book- Prentice Hall. By David Van den Bout

[7] VHDL Starter's Guide.-Prentice Hall. By Sudhakar Yalamanchili

[8] Data Acquisition Linear devices Data Book - National Semiconductor.

[9] Digital Designing with Programmable Logic Devices.-Prentice Hall.By John W. Carter

[10] International Journal of Advanced Research in Computer Science and Software Engineering (Volume 3, Issue 8, August 2013)

[11] Programmable Logic Design Quick Start Guide (UG500 (v1.0) May 8, 2008)

[12] V. Jayaprakasan1,M.Madheswaran, "FPGA Implementation of FIR based Decimation Filter Structure for WiMAX Application", International Journal of Advanced Research in Computer and Communication Engineering Vol. 2, Issue 7, July 2013

[13] Gaurav Sharma, Arjun Singh Chauhan, Himanshu Joshi, "Delay Comparison of 4 by 4 Vedic Multiplier based on Different Adder Architectures using VHDL", International Journal of IT, Engineering and Applied Sciences Research (IJIEASR) ISSN: 2319-4413 Volume 2, No. 6, June 2013

\section{BIOGRAPHIES}

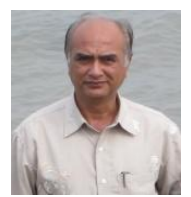

Born in May 1960, Dr. Haresh N. Pandya Professor \& Head, Department of Electronics, Saurashtra University, Rajkot, Gujarat, India. I obtained B.Sc., M.Sc. and Ph.D. degrees from Saurashtra University-Rajkot. He specialized in the area of Electronic circuits and devices. My Research area in Microprocessor, Microcontroller, Embedded System \& Robotics. Published 78 numbers of technical papers in deferent international journals.

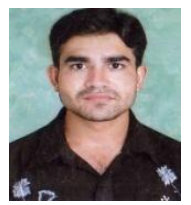

Born in December 1986, Mr. Mahesh Rangapariya is currently working as a Ph.D. Student \& Visiting Lecturer at Department of Electronics, Saurashtra University-Rajkot. I obtained Master's Degree in ELECTRONISC from Department of Electronics Saradar Patel University-Vallabh Vidhaya Nagar. Attended Two-day "WOKRSHOPE ON EMBEDDED SYSTEMS" at Department of Electronics Saradar Patel University-Vallabh Vidhaya Nagar 2009. Attended One-day "WOKRSHOPE ON EMBEDDED SYSTEMS" at The Institution of Electronics and Telecommunication Engineers (IETE)-Rajkot 2010, he obtained B.Sc. degree in electronics from Gujarat University Ahmadabad in 2007.

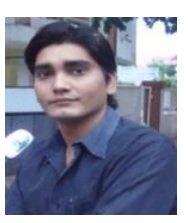

Born in July 1986, Mr. Jitendra Rajput is currently working as Assistant professor of Electronics at SSR College of Science,(Affiliated to Pune University), Silvassa- 396230, D \& NH, India. I obtain Master's Degree in ELECTRONISC from Department of Electronics Saradar Patel University-Vallabh Vidhaya Nagar. Attended Two-day "WOKRSHOPE ON EMBEDDED SYSTEMS" at Department of Electronics Saradar Patel University-Vallabh Vidhaya Nagar 2009. Attended Two-day "WOKRSHOPE ON EMBEDDED SYSTEMS" at Microsystems LTD., Chennai. Held during March 23-24, 2010. The workshop included comprehensive module on Embedded Processor, Architecture, Applications and Real Time Operating System (RTOS). 